\title{
Recruitment and Retention of African American Patients for Clinical Research: \\ An Exploration of Response Rates in an Urban Psychiatric Hospital
}

\author{
Estina E. Thompson \\ Institute for Social Research University of Michigan \\ Harold W. Neighbors \\ Institute for Social Research University of Michigan \\ Cheryl Munday \\ Department of Psychology Detroit Psychiatric Institute \\ James S. Jackson \\ Institute for Social Research University of Michigan \\ ABSTRACT
}

\begin{abstract}
The issues related to recruiting African American psychiatric inpatients are discussed in the context of a study on the influence of ethnicity on psychiatric diagnosis. Ethnically diverse psychiatric residents interviewed 960 Black and White inpatients in 2 urban psychiatric hospitals. Despite the obstacles cited in the literature about recruiting and retaining African Americans into research, $78 \%$ of this sample were African American. In addition, interview completion and refusal rates did not differ by patient ethnicity. Results suggest that matching interviewer and patient ethnicity did not influence African Americans' likelihood of participating in or of refusing an interview. This article summarizes a number of guidelines that others may find useful in conducting clinical research with African Americans, ranging from the formation of academic_-public liaisons to interviewer training.
\end{abstract}

This article is based on data collected by the African American Mental Health Research Center (AAMHRC), which is affiliated with the Program for Research on Black Americans, directed by James S. Jackson and located at the University of Michigan's Institute for Social Research.

This study would not have been possible without the support and guidance of the Detroit Psychiatric Institute (DPI) and the cooperation of Sinai Hospital. We thank all of the DPI and Sinai Hospital staff who worked to make this project a success, and we note the special contributions of Lawrence Jackson, Director of the Detroit Psychiatric Institute; Walter Swoboda and Linda Hotchkiss of Sinai Hospital; and Victoria Binion and Arnold Greenfield of the Michigan Department of Mental Health. We thank the members of the AAMHRC National Advisory Panel (Carl Bell, A. J. Franklin, Linda K. George, Mary Schwab-Stone, M. Belinda Tucker, Sam Turner, and Donald Williams) for their expertise and advice. 
We also thank the interviewers and especially the respondents who agreed to be interviewed for this study. This project was funded by the Michigan Department of Mental Health and the National Institute of Mental Health.

Correspondence may be addressed to Harold W. Neighbors, Program for Research on Black Americans, Institute for Social Research, University of Michigan, Room 5006, RCGD-ISRI, Ann Arbor, Michigan, 48106-1248.

Received: July 15, 1995

Revised: November 29, 1995

Accepted: December 1, 1995

It is generally agreed that African Americans are at increased risk for the development of serious mental health problems ( Williams, 1995 ). The Community Mental Health Movement's emphasis on eliminating inequities in access to treatment has made African Americans a priority for mental health service delivery for the past 35 years ( Wagenfeld \& Jacobs, 1982; Neighbors, 1987). However, despite policies focused on African Americans, scholars still argue that inadequate attention to ethnic and cultural differences has resulted in the inability of traditional mental health services to meet their emotional needs (Lukoff, Lu, \& Turner, 1992 ; Snowden, 1993; Sue, 1992; Williams, 1995 ). As a result, many have called for more and better clinical research on such issues as stigma, the institutionalization process, access to outpatient care, the premature termination of treatment, and diagnosis ( Cannon \& Locke, 1977 ; Harrison, Jackson, Munday, \& Bleiden, 1988 ).

When reviewing the clinical research literature on African Americans, one cannot help but notice a fair amount of pessimism surrounding the ability of researchers to recruit and retain adequate numbers of African American patients. For example, Neal and Turner's (1991) review of research on anxiety disorders among African Americans prompted the following statement:

Throughout this article we have emphasized the need for further research in the area of anxiety disorders among African Americans. A frequent comment heard among our colleagues is 'We would love to do the research but we just can't get the subjects.'(p. 408)

There can be no doubt that over the past 20 years, the number of African American researchers, as well as the number of articles published on clinical issues regarding African Americans, has increased substantially ( Lawson, 1986; Neighbors et al., 1992 ). However, data collections with African Americans in clinical settings still suffer from inadequate descriptions of how to successfully plan and execute such studies. Too often, researchers focus on substantive findings without discussing the practical details of the procedures used to recruit and retain African American patients ( Locke, 1977 ; Lawson, 1986 ; Neal \& Turner, 1991 ).

The purpose of this article is to address some of the gaps identified in the literature by describing the methods used for a large-scale clinical study of psychiatric inpatients conducted by the University of 
Michigan's African American Mental Health Research Center (AAMHRC). The study was motivated by our interest in exploring ethnic and cultural influences on psychiatric diagnostic processes ( Neighbors, Jackson, Campbell, \& Williams, 1989). Future articles will provide a more in-depth investigation of differences between African Americans and Whites in diagnostic processes and outcomes. The present discussion describes the data collection procedures used in a clinical study conducted in a low-income, urban setting. It provides an account of the practical issues that were addressed while planning and implementing a collaborative effort between university-based researchers and clinical staff at a public psychiatric hospital. This article is intended to provide a guide for researchers interested in conducting similar clinical investigations of severely mentally ill African Americans. The results presented here clearly show that, although it may be difficult to recruit adequate numbers of African American patients for clinical research, it is not impossible. This is especially the case if the researchers use tried and proven data collection techniques that can be borrowed and transformed from community-based survey research studies ( Jackson, Tucker, \& Bowman, 1982 ; Milburn, Gary, Booth, \& Brown, 1991 ).

Evaluating the effectiveness of any data collection effort with African Americans has to begin with an assessment of response rates. Low response rates seriously limit the representativeness of the data, and they impede the capability of conducting multivariate statistical analyses. Specific recommendations for ethnic minority research include the use of a cross-cultural research perspective, intensive preliminary interaction with African American community members, immersion in the local environment to increase community "ownership" of the research project, African American interviewers, intensive interviewer training and preparation, and extensive pretesting of instruments as well as data collection procedures ( Jackson et al., 1982 ). Following these methods can lead to encouraging data collection results. Here we describe how these research guidelines were adapted to a psychiatric hospital predominantly serving African American and White mentally ill patients.

\section{Obstacles to Clinical Research With African Americans}

Institutional racism has been a major impediment to clinical research on African Americans ( Thomas \& Sillen, 1972 ). Historically, the question of African American mental health has been used politically as a justification for slavery, as a "scientific" rationale for segregation, and to reinforce the concept of African American racial inferiority ( Williams, 1986). Hirsch (1981) and Kamin (1974) showed that some researchers either misrepresented their work or, in some cases, falsified data (Lawson, 1986, p. 50 ). This type of work has not only fostered negative images of African Americans, it has also made it difficult to recruit African American patients into research studies. Understandably, African Americans tend to distrust research in general and research conducted by Whites in particular. Researchers are viewed as taking from the community and not giving anything back, which is often the result of research scientists being insensitive to special issues related to ethnicity and culture ( Cross, Bazron, Dennis, \& Isaacs, 1989 ; Ladner, 1973 ). In addition to the high level of distrust toward research, the stigma that some African Americans attach to the concepts of mental health and illness makes participation that much more problematic ( Griffith \& Baker, 1993). As a result, sufficient time and money should be allocated during the research planning stage to develop culturally sensitive strategies that incorporate the 
reactions of African American patients into the preparation and implementation process.

Another problem in doing clinical research with African Americans is the negative attitudes toward psychotherapy that exists within the community. Many African Americans with emotional problems do not seek help from mental health services but, instead, rely on a number of alternative help resources including ministers, primary care physicians, and informal support networks ( Neighbors, 1988), or they seek no outside help at all ( Neighbors \& Jackson, 1984). This often results in inadequate pools of African Americans from which to sample. Many African Americans who do enter the formal mental health system terminate their treatment prematurely ( Neighbors et al., 1992; Spurlock, 1988, p. 32). African American patients from inner-city, low-income areas may be especially reluctant to participate in research studies in major medical centers ( Neal \& Turner, 1991, p. 401). As a result, African American patients are frequently considered to be a research risk because they are less likely to be found or to participate for the duration of the study. The mistaken notion that African American patients cannot understand the research design or follow the procedures also contribute to the lack of participants for research ( Spurlock, 1988 ). Finally, African American men are sometimes viewed as violent and threatening and, thus, not good candidates for research. These factors undoubtedly contribute to the fact that many researchers may be reluctant to conduct research focused specifically on African Americans.

This project relied on four major components of an overall research strategy designed to ensure that adequate numbers of African American patients would be enrolled in the study. The foundation of this project was the formation of a working relationship between an academically based research center and a "front-line" mental health service delivery site. This partnership resulted in combining the strengths of survey research methodology with clinical expertise, which increased both institutions' ability to compete for research funding. Having access to an urban clinical site for research was also instrumental in the ability to successfully recruit and retain sufficient numbers of African American psychiatric patients.

The second component was interviewer recruitment and selection. This project needed a pool of interviewers that had previous clinical experience working with severely mentally ill patients in general and low-income African American inpatients in particular. Prior experience with this target group minimized problems that can arise when attempting to study patients who are considered "hard to reach."

The third component of the strategy was to concentrate on providing extensive and consistent training across interviewers. Interviewers practiced how to approach patients, how to provide a general overview of the study, and the best method of presenting topics such as confidentiality and voluntary participation. They also were required to demonstrate an understanding of the intricacies of the survey instruments that would be used during the actual data collection. Such thorough prestudy training reduces the likelihood of nonresponse.

The fourth component of the study was to begin to address issues of cultural sensitivity. This was done, indirectly, by exploring the impact of ethnicity on important mental health processes. One important aspect of cultural sensitivity is the idea of matching client and therapist according to obvious similarities such as skin color or ethnic group membership. Matching has attracted interest because it is relatively 
easy to operationalize, to the extent that the investigators can successfully recruit sufficient numbers of multiethnic patients and clinicians. Matching (and by implication, mismatching) can also indirectly test the impact of cultural sensitivity. The assumption here is that two individuals who are members of the same ethnic group are likely to share similar cultural backgrounds and, as a result, will experience a higher degree of understanding and communication than two people from dissimilar backgrounds ( Anderson \& Carter, 1982; Griffith, 1977 ; Ridley, 1984 ).

One goal of the present study was to empirically test the impact of matching interviewers and patients on such issues as diagnosis and patient recruitment. It is reasonable to assume that patients approached by ethnically similar clinicians would be more likely to participate in research than patients approached by ethnically dissimilar clinicians. It is important to note that no specific efforts were made to train interviewers to be culturally sensitive. Such an intervention would have made it more difficult, if not impossible, to assess the degree to which sharing similar backgrounds and experiences does result in positive outcomes ( Neighbors, Braithwaite, \& Thompson, 1995, p. 286). The point, however, is that because we used clinicians and patients who were African American-as well as those who were not-it is possible to empirically determine the impact of ethnic matching on study participation. In summary, each of these four components of the research plan were important in recruiting and retaining African American psychiatric inpatients for clinical research.

The problems inherent in doing clinical research with African American patients are not unlike the barriers that social scientists have confronted in doing community-based survey research in African American communities. One positive result arising from these community research experiences is the conclusion that it is possible to obtain high levels of participation from African Americans in these studies. It is our position that a number of the recommendations for community research can be translated to clinical settings. Despite the ever-present obstacles and barriers to conducting research with African Americans, if the research team is willing to follow a few well-documented guidelines, adequate numbers can be recruited into clinical research studies.

\section{Method}

\section{Research Sites}

Two hospitals located in Detroit, Michigan, were selected as sites for the study. 1 The Detroit Psychiatric Institute (DPI), a 148-bed state psychiatric facility, and Sinai Hospital, a private hospital containing a 37bed psychiatric ward. The DPI patient population contained 60\%-65\% African Americans and $58 \%-61 \%$ men, the majority of whom were indigent and involuntarily admitted. Sinai's patient population was typically 50\%-60\% African American and 40\% male.

\section{Interviewer Selection and Training}

Although an appropriate study site is necessary to gain access to the population of interest, it is equally important to have a sufficient number of interviewers who are highly trained and who possess 
characteristics known to have a positive influence on study outcomes. Interviewers had to have adequate clinical experience with low-income African American patients. The research staff felt that this was essential to successfully recruit patients into this study. Thus, applicants for the interviewing positions were screened for their ability to work with patients similar to the study population. In addition, sufficient numbers of African American interviewers were needed to permit an assessment of the effect of interviewer ethnicity on the recruitment of African American patients.

Research interviewers were recruited from the Wayne State University psychiatric residency programs at DPI, University Psychiatric Center (Lafayette Clinic), and Sinai Hospital. The project was discussed with the respective directors of residency training, and their approval to recruit residents was obtained. Flyers were posted and distributed to residents. Presentations were made to groups of residents to increase the applicant pool. Residents who expressed an interest in the study were individually interviewed by a clinical psychologist (the on-site clinical coordinator) who served as an integral part of the research team. As the study progressed, there was some attrition as residents graduated and left the area. Additional recruiting efforts included contacting recent graduates of the residency programs and communicating with professional organizations such as the Association of Black Psychiatrists and the Michigan Psychiatric Society.

A total of 15 third- and fourth-year psychiatric residents (African American, White, Middle Eastern, and African) served as interviewers over the course of the study. Interviewers were carefully trained on how to approach patients and introduce the study. They were also trained extensively on procedures for obtaining written patient consent. Interviewers were instructed not to interview any patient who, they believed, neither understood the study's intent nor was physically or mentally able to be interviewed. Interviewers were also told that they could terminate an interview if they were concerned about their safety. Patients were told that they could terminate an interview at any time for any reason without losing the financial compensation promised. Eligible patients were paid $\$ 5$ for agreeing to participate in the study.

Interviewers recorded the study outcomes in a logbook. Seven result codes were created to distinguish reasons for sample attrition: (a) completed, (b) partial interview because the respondent prematurely terminated the interview, (c) no interview because the patient was discharged before contact, (d) no interview because the patient refused to be interviewed or sign the consent form, (e) no interview because the patient was incapable of being interviewed, (f) no interview because the interviewer knew the respondent, and $(\mathrm{g})$ the interviewer did not report the interview's disposition. These result codes form the basis of the analyses presented here.

\section{Results}

Newly admitted African American and White adult inpatients with an admitting diagnosis of a schizophrenic or mood disorder were eligible to participate in the study. Over the entire study, approximately $62 \%$ of the eligible patients were male, $59 \%$ had an admitting diagnosis of a schizophrenic disorder, and 78\% were African American. The percentage of men, African Americans, 
and patients with a schizophrenic disorder who were eligible for an interview at each site approximated their proportions in the respective hospitals. A total of 961 patients completed interviews, $78 \% n=752$ of whom were African American. $\underline{2}$

The findings presented in this article focus on differences between African Americans and Whites in study participation and reasons for nonresponse, controlling for the patient's admitting diagnosis. Particular attention is paid to highlighting patient refusal rates in comparison with early discharge as reasons for nonresponse. The refusal rate most accurately reflects a patient's decision to participate. In this study, the decision to participate (or to refuse) is the direct result of the face-to-face meeting between the patient and the interviewer. It is at this point that the patient is introduced to the study and invited to be interviewed. If the patients are not approached in a courteous and professional manner, they most likely will refuse. Therefore, it is crucial in studies of this type that the interviewing staff is not only well trained but experienced in working with clients from a variety of ethnic backgrounds. In short, both the interview completion and the refusal rates reflect the individual characteristics of the patient (degree of comfort, willingness to participate, mistrust of research, compensation) and the interviewer (level of training, presentation expertise, sensitivity to cultural differences), as well as the interaction between the two (e.g., ethnic match).

As stated earlier, clinical researchers have consistently cited problems with recruiting African American patients for research. The implication for the present study is that they should be more likely than White patients to refuse to participate in the study and less likely to complete an interview. Table 1 shows the relationship between patient ethnicity and study participation across both project sites. The interview completion rate for the entire patient sample was 56\%. Table 1 also shows that there were no differences between African American and White patients in the interview completion rate, the refusal rate, or the rate of early discharge.

Ethnic matching between interviewers and eligible respondents has been proposed as one possible solution to increasing African American participation in clinical research ( Neighbors et al., 1992 ). The literature on ethnic matching implies that African American patients would be more likely to participate in research and less likely to refuse an interview when approached by an African American interviewer versus an ethnically different interviewer. To address possible differences in the needs or preferences among the African American patients, we examined their reasons for nonresponse by admitting diagnosis and interviewer ethnicity. Table 2 shows that matching African American patients with African American interviewers did not influence interview completion rates. Completion rates were slightly higher for patients with schizophrenia than for those with mood disorders. Similarly, matching exerted no noticeable influence on refusal rates. It appears that patient diagnosis played a far more important role in the likelihood of not responding. African American patients with an admitting diagnosis of schizophrenia were much more likely to refuse to participate than African American patients with depression, regardless of the ethnic match condition. It is interesting to note that the lower refusal rates among the mood disorder patients might have been influenced by the relatively high early release rate among this group. Unfortunately, we do not know how many of these discharged depressed patients would have participated if interviewers had been able to introduce them to the study. 


\section{Discussion}

The findings reported here suggest that a patient's admitting diagnosis was a more influential factor in the patterns of nonresponse than in completing interviews. It is encouraging to note that interview completion rates were between $53 \%$ and $59 \%$ for each Ethnicity $\times$ Diagnosis group. $\underline{3}$ Patients with schizophrenia were more likely to refuse to be interviewed, whereas patients with depression were more likely to leave the hospital before being introduced to the study. Certainly one would expect a higher refusal rate among those with a diagnosis of schizophrenia where psychotic symptoms might be more prominent. For example, patients who are disoriented, agitated, confused, or highly suspicious might be less likely to respond to the interviewers' invitation to participate. In contrast, depression is a disorder characterized by disturbance of mood and affect with less disruption of cognition or gross impairment of reality testing. More detailed analyses of these data and additional information from the patients' medical charts should help to clarify the results reported here, particularly why such a large proportion of eligible African American patients with depression were discharged before interviewers were able to contact them.

Although the admitting diagnosis played a significant role in response patterns, matching interviewers and patients on the basis of ethnicity did not have much of an impact. It is not clear at this point why matching was not related to participation. The effects of matching could have been diluted by the heavy emphasis placed on interviewer selection and training. For example, both African American and White interviewers were selected on the basis of their experience in working with low-income patients from urban settings. Similarly, both groups were exposed to the same training procedures for introducing the study and eliciting the cooperation of patients. Particular emphasis was placed on techniques for encouraging reluctant patients to participate. Because the interviewers were also psychiatric residents at the research sites, they were able to make repeated callbacks to patients who were initially unsure about participating. It may be that the effects of matching are more important for variables such as mental health service use, diagnosis, length of stay, or treatment effectiveness than for recruitment into research per se ( Neighbors et al., 1992 $)$. The point, however, is that with ample preparation, both African American and White clinical interviewers can successfully interact with patients from a variety of ethnic backgrounds. This is useful because African American clinical researchers are still relatively rare ( Cannon \& Locke, 1977 ; Neal \& Turner, 1991 ). To the best of our knowledge, no study has investigated the effect of patient-interviewer ethnic matching on Refusal Rates $\times$ Patient Diagnosis in such a large sample $n=752$ of African American patients. As a result, these findings need to be replicated in other settings.

The results presented here clearly show that with careful planning, substantial numbers of severely mentally ill African American inpatients can be recruited to participate in clinical research. The success of this study can be attributed to a number of guidelines others may find useful in conducting clinical research with African American patients. The research staff must have extensive prestudy interaction with key institutional personnel. Such individuals are analogous to the "key informants" or "gatekeepers" that are so instrumental in community research. In the present study, research staff collaborated with DPI 
clinical staff during the writing of the proposal. Thus, when the study was funded, key hospital staff, including the director of the hospital, had already indicated their support for the data collection. The onsite clinical coordinator served as the main point of contact and was the key insider who provided critical information and advice on all aspects of the study. Once the initial plans had been completed, the research staff presented the general design of the study to relevant hospital department heads. This proved extremely useful in ensuring the cooperation of the necessary personnel. In short, to conduct studies of this type, it is necessary to take adequate time before actual data collection to implement a nonobtrusive set of procedures that will blend with the ongoing hospital operations.

It is important to devote ample time to pilot work and pretesting. The automated interview scheduling and monitoring system that was developed specifically for this study was constructed on the basis of obstacles encountered while testing preliminary data collection procedures. This computer program proved to be essential for ensuring a smooth and efficient method for linking eligible patients with available interviewers once the actual study began. It not only kept track of a patient's interview status but also reduced the number of errors made when determining patient eligibility. The monitoring database ensured that no patient who had completed or refused an interview was mistakenly interviewed again, even among those who were released and later readmitted to DPI. In addition, the program generated regular progress reports of completed interviews and reasons for noninterviews that, in turn, identified problems with the data collection procedures or with specific interviewers. Using a computerized database for monitoring field procedures was essential in managing the substantial number of clinicians and their changing schedules.

It is important to use qualified and well-trained interviewers who are "indigenous" to the study site. Our options were somewhat limited in the sense that a clinically trained interviewing staff had to be used. Nevertheless, we were able to use interviewers who were familiar with DPI and Sinai and, therefore, knowledgeable about hospital protocol. This decreased possible conflicts between study and hospital staff and facilitated communication between the hospital staff and the interviewers. More important, however, was the extensive training conducted with the interviewing staff, including providing an introduction to the study and the overall research objectives. During training, the interviewers were exposed to a line-by-line, page-by-page review of the instructions for introducing the study as well as all interviewing protocols. This included mandatory role playing practice interviews with each other and the completion of two practice interviews with real patients. These practice interviews were reviewed and critiqued by the clinical coordinator. Practice sessions also included rehearsing how to introduce and explain the study to the patients. The primary research staff provided a recommended script that interviewers could modify.

The research instruments used in the present data collection were extensively tested, first among the primary research staff and later with postdoctoral trainees based at the data collection site. After interviewing at least 2 or 3 patients, these postdoctoral scholars provided extensive feedback to the study staff. These evaluations were critical in deciding which instruments would be used and, once chosen, what modifications to make to them. For example, skip patterns and interviewer checkpoints were added to one of the instruments, the Diagnostic and Statistical Manual of Mental Disorders (3rd ed., rev.; $D S M-I I I-R$ ) Symptom Checklist, to make it easier to follow. It was also at this point that interviewers 
suggested that sample language be added that could be used as an aid in probing positive symptoms. Wording from other diagnostic instruments such as the Schedule for Affective Disorders and Schizophrenia, the Diagnostic Interview Schedule (DIS), the Composite International Diagnostic Interview, and the Structured Clinical Interview for $D S M-I I I-R$ (SCID) was used for this purpose. The result was a structured, yet flexible, guide for pursuing a $D S M-I I I-R$ ( $\underline{\text { American Psychiatric }}$ Association, 1987 ) diagnosis.

Instrument development was also crucial because of the nature of the study population. These patients were predominantly low-income African Americans who, as the literature has documented, had very little (if any) experience participating in clinical research. A review of a number of diagnostic research instruments (including the DIS and the SCID) and subsequent pretesting with each one revealed variability with respect to interview length and respondent burden. While initial plans were to use the DIS, previous studies had shown that this highly structured instrument was somewhat problematic when used with a similar, predominantly African American patient population ( Hendricks, Bayton, et al., 1983 ). Our pretest results also indicated that a highly structured instrument like the DIS was difficult to use with severely compromised patients. As a result, a more flexible approach to diagnostic assessment was used in this study.

The results presented here demonstrate that large-scale data collection in psychiatric settings with substantial numbers of African Americans is feasible. It is particularly important that more studies of this type compute response rates. Not reporting such important descriptive statistics may leave the impression that the researchers did not encounter difficulties in collecting clinical data. Conversely, the lack of response statistics could be contributing to an overly negative impression with respect to recruiting minorities for clinical research. For example, Miranda, Azocar, Organista, Munatoz, and Lieberman (1996) show that, by relying on culturally sensitive face-to-face screening and recruitment methods, extremely high response rates can be attained for Latinos in clinical trials. The results presented here are also encouraging. The present study was able to obtain over 700 successfully completed interviews with African Americans. Furthermore, short length of stay and noninterviews for other reasons (e.g., illness, inadequate logging procedures) contributed as much to nonresponse as did patient refusals. Thus, it is also important that clinical researchers delineate reasons for nonresponse.

The impetus for this project was generated in 1988 when representatives from the National Institute for Mental Health, the Michigan Department of Mental Health, the University of Michigan, and the DPI convened a mental health research conference entitled, "A Search for Understanding" ( Harrison et al., 1988 ). This conference represented one of the first occasions for university-based researchers and public mental health clinicians to discuss a number of important mental health issues facing African Americans ( Bell, 1988; Neighbors, 1988 ). This study demonstrates the importance of establishing collaborative research relationships between university-based researchers and public mental health facilities. These partnerships provide opportunities for scholars in each setting to investigate issues in a manner not possible were they working alone. Very often, clinicians on the frontlines develop hypotheses about clinical processes that they find difficult to investigate because of time constraints and lack of financial resources. University-based researchers, on the other hand, although having the time and resources to 
generate funding for research often do not have access to the clinical knowledge or to the populations necessary to address the kinds of problems encountered by African American inpatients.

In conclusion by focusing on the practical issues of clinical research with African Americans we have been able to provide more detail about how to prepare for the inevitable challenges of hospital-based data collection. We felt that it was important to focus on the practical issues regarding study procedures because recruitment issues are important for minority mental health research and because it provides a thorough basis for subsequent articles. We hope that this report on our experiences serves as an example that will encourage more collaborative studies between universities and public mental health facilities. Collaborative research projects of this nature can help solve some of the problems facing ethnic minorities having clinical contact with the mental health service delivery system.

\section{References}

American Psychiatric Association. Diagnostic and statistical manual of mental disorders (3rd ed., rev.). Washington, DC: Author.

Anderson, L. \& Carter, J. H. (1982). Psychotherapy: Patient—therapist matching reconsidered.( Journal of the National Medical Association, 74, 461-464.)

Bell, C. C. (1988). Needed research in assessment and diagnosis.(In A. O. Harrison, J. S. Jackson, C. Munday, \& N. B. Bleiden (Eds.), Asearch for understanding: The Michigan Research Conference on Mental Health Services for Black Americans (pp. 26-31). Detroit, MI: Wayne State University Press.) Cannon, M. S. \& Locke, B. Z. (1977). Being Black is detrimental to one's mental health: Myth or reality? Phylon, 38, 408-428. (Cross T. L. Bazron B. J. Dennis K. W. Isaacs M. R. 1989 Washington, DC: Child and Adolescent Service System Program Technical Assistance Center, Georgetown University Child Development Center.)

Griffith, E. H. \& Baker, F. M. (1993). Psychiatric care of African Americans.(In A. C. Gaw (Ed.), Culture, ethnicity and mental illness (pp. 147-173). Washington, DC: American Psychiatric Press.) Griffith, M. S. (1977). The influences of race on the psychotherapeutic relationship.( Psychiatry, 40, 27-40.)

Harrison, A. O., Jackson, J. S., Munday, C. \& Bleiden, N. B. (Eds.). (1988). A search for understanding: The Michigan Research Conference on Mental Health Services for Black Americans. (Detroit, MI: Wayne State University Press)

Hendricks, L. E., Bayton, J. A., Collins, J. L., Mathura, C. B., McMillian, S. R. \& Montgomery, T. A. (1983). The NIMH Diagnostic Interview Schedule: A test of its validity in a population of Black adults.( Journal of the National Medical Association, 75, 667-671.)

Hirsch, J. (1981). To unfrock the charlatans.( Sage Race Relations Abstracts, 6, 1-65.)

Jackson, J. S., Tucker, M. B. \& Bowman, P. J. (1982). Conceptual and methodological problems in survey research on Black Americans.(In W. T. Liu (Ed.), Methodological problems in minority research (Occasional Paper No. 7, pp. 11-39). Chicago: Pacific/Asian American Mental Health Research Center.)

Kamin, L. J. (1974). The science and politics of IQ. (Hillsdale, NJ: Erlbaum) Ladner, J. (1973). The death of White sociology. (New York: Vintage Books) 
Lawson, W. B. (1986). Racial and ethnic factors in psychiatric research.( Hospital and Community Psychiatry, 37, 50-54.)

Lukoff, D., Lu, F. \& Turner, R. (1992). Toward a more culturally sensitive DSM-IV: Psychoreligious and psychospiritual problems.( Journal of Nervous and Mental Disease, 180, 673-682.)

Milburn, N. G., Gary, L. E., Booth, J. A. \& Brown, D. R. (1991). Conducting epidemiologic research in a minority community: Methodological considerations.( Journal of Community Psychology, 19, 3-12.) Miranda, J., Azocar, F., Organista, K. C., Muñoz, R. \& Lieberman, A. (1996). Recruiting and retaining low-income Latinos in psychotherapy research.( Journal of Consulting and Clinical Psychology, 64, 868-874.)

Neal, A. M. \& Turner, S. M. (1991). Anxiety disorders research with African Americans; Current status.( Psychological Bulletin, 109, 400-410.)

Neighbors, H. W. (1987). Improving the mental health status of Black Americans: Lessons from the Community Mental Health movement.( Milbank Memorial Fund Quarterly, 65, 348-380.) Neighbors, H. W. (1988). Needed research on the epidemiology of mental disorders in Black Americans.(In A. O. Harrison, J. S. Jackson, C. Munday, \& N. B. Bleiden (Eds.), A search for understanding: The Michigan Research Conference on Mental Health Services for Black Americans (pp. 49-60). Detroit, MI: Wayne State University Press.)

Neighbors, H. W., Bashur, R., Price, R., Selig, S., Donabedian, A. \& Shannon, G. (1992). Ethnic minority mental health service delivery: A review of the literature.( Research in Community and Mental Health, 7, 55-71.)

Neighbors, H. W., Braithwaite, R. \& Thompson, E. (1995). Health promotion and African Americans: From personal empowerment to community action. ( American Journal of Health Promotion, 9, 281-287.)

Neighbors, H. W. \& Jackson, J. S. (1984). The use of informal and formal help: Four patterns of illness behavior in the Black community.( American Journal of Community Psychology, 12, 629-644.) Neighbors, H. W., Jackson, J. S., Campbell, L. \& Williams, D. (1989). The influence of racial factors on psychiatric diagnosis: A review and suggestions for research.( Community Mental Health Journal, 25, 301-311.)

Ridley, C. R. (1984). Clinical treatment of the nondisclosing Black client.( American Psychologist, 39, 1234-1244.)

Snowden, L. (1993). Emerging trends in organizing and financing human services: Unexamined consequences for ethnic minority populations.( American Journal of Community Psychology, 21, 1-13.) Spurlock, J. (1988, April 18-19). Needed research in treatment.(In A. O. Harrison, J. S. Jackson, C. Munday, \& N. B. Bleiden (Eds.), Asearch for understanding: The Michigan Research Conference on Mental Health Services for Black Americans. Detroit, MI: Wayne State University Press.)

Sue, S. (1992). Ethnicity and mental health: Research and policy issues.( Journal of Social Issues, 48, 187-208.)

Thomas, A. \& Sillen, S. (1972). Racism and psychiatry. (New York: Brunner/Mazel)

Wagenfeld, M. \& Jacobs, I. (1982). The Community Mental Health Movement: Its origin and growth.(In M. Wagenfeld, P. Lemkau, \& B. Justice (Eds.), PublicMental Health (pp. 46-88). Beverly Hills, CA: Sage.)

Williams, D. (1986). The epidemiology of mental illness in Afro-Americans.( Hospital and Community Psychiatry, 37, 42-49.) 
Williams, D. (1995). African American mental health: Persisting questions and paradoxical findings.(In R. Taylor (Ed.), African American research perspectives (pp. 8-16). Ann Arbor, MI: Program for Research on Black Americans, African American Mental Health Research Center, Institute for Social Research.)

\section{1}

All descriptive data concerning the hospitals' composition and capacity apply to the time of the study. The results presented in Tables 1 and $\underline{2}$ collapse across research settings.

\section{2}

One of the 961 patients was classified as Latino. This respondent was excluded from the analyses presented in Tables 1 and $\underline{2}$.

\section{3}

The completion rate was 59\% for African American patients with an admitting diagnosis of schizophrenia, 53\% for African American patients with mood disorder, 57\% for White patients with schizophrenia, and $53 \%$ for White patients with mood disorder.

Table 1.

\begin{tabular}{|c|c|c|c|c|c|c|c|c|c|}
\hline \multicolumn{10}{|l|}{$\begin{array}{l}\text { ]able I } \\
\text { Ratioust ior Fir. }\end{array}$} \\
\hline \multirow[b]{2}{*}{ Patietul ethnicity } & \multicolumn{2}{|c|}{ completed" } & \multicolumn{2}{|c|}{ Reruss } & \multicolumn{2}{|c|}{ Ditithidged } & \multicolumn{2}{|c|}{ Other } & \multirow[b]{2}{*}{ is } \\
\hline & $n$ & Raw L & il & Kow & भ & Row & $n$ & Rum & \\
\hline Black & 742 & 5 & 21111 & 15 & 274 & 17 & 156 & 1.2 & 1,733 \\
\hline White & $21 \%$ & 50 & i. 5 & 17 & 50 & 13 & 59 & 15 & 382 \\
\hline Io1:al & 960 & 56 & 266 & 16 & 274 & 1.4 & 215 & 13 & 1,715 \\
\hline
\end{tabular}

Table 2. 
$<$ I $>$ Towards a culturally competent system of care. $\langle/ \mathrm{I}\rangle$

Iat:la: 2

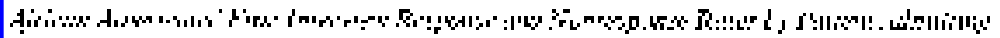

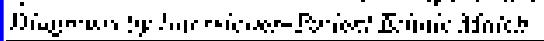

\begin{tabular}{|c|c|c|c|c|c|c|c|c|c|}
\hline \multirow[b]{2}{*}{ 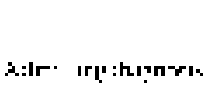 } & \multicolumn{2}{|c|}{ 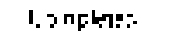 } & \multicolumn{2}{|c|}{.2ertuk } & \multicolumn{2}{|c|}{ Lusthingd } & \multicolumn{2}{|c|}{ C.i=1 } & \multirow[b]{2}{*}{3} \\
\hline & $s$ & $k r=3$ & $s$ & Lix & $\therefore$ & sous & $"$ & $R_{n} \times 6$ & \\
\hline \multicolumn{10}{|c|}{ 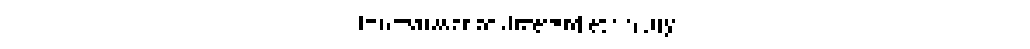 } \\
\hline 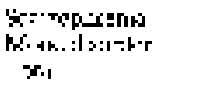 & $\begin{array}{l}211 \\
1 \mathrm{n} \\
34\end{array}$ & $\begin{array}{l}i_{1} \\
i 1 \\
5 !\end{array}$ & به: & $\begin{array}{l}18 \\
\vdots: \\
15\end{array}$ & $\begin{array}{l}31 \\
x\end{array}$ & 11 & $\begin{array}{l}4: \\
\vdots \vdots \\
\vdots \\
\vdots\end{array}$ & ימין & $\begin{array}{c}\because \vdots \\
\vdots i \\
\vdots 1\end{array}$ \\
\hline \multicolumn{10}{|c|}{ 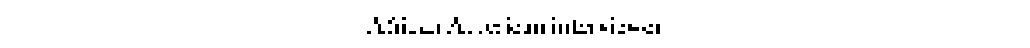 } \\
\hline 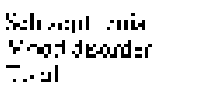 & $\begin{array}{l}\text { "n } \\
1+n \\
+41\end{array}$ & 3 & $\begin{array}{l}\text { y. } \\
12 \\
1\end{array}$ & 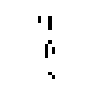 & ה.1. & $\begin{array}{l}\because \\
i \\
i\end{array}$ & $\begin{array}{l}13 \\
11 \\
\because\end{array}$ & $\begin{array}{l}1.2 \\
1.2\end{array}$ & 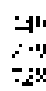 \\
\hline
\end{tabular}


Table 1

Reasons for First Interview Response and Nonresponse Within Each Patient Ethnicity

\begin{tabular}{|c|c|c|c|c|c|c|c|c|c|}
\hline \multirow[b]{2}{*}{ Patient ethnicity } & \multicolumn{2}{|c|}{ Completed $^{\mathrm{a}}$} & \multicolumn{2}{|c|}{ Refused } & \multicolumn{2}{|c|}{ Discharged } & \multicolumn{2}{|c|}{ Other } & \multirow[b]{2}{*}{$N$} \\
\hline & $n$ & Row $\%$ & $n$ & Row $\%$ & $n$ & Row $\%$ & $n$ & Row \% & \\
\hline Black & 752 & 56 & 201 & 15 & 224 & 17 & 156 & 12 & 1,333 \\
\hline White & 208 & 55 & 65 & 17 & 50 & 13 & 59 & 15 & 382 \\
\hline Total & 960 & 56 & 266 & 16 & 274 & 15 & 215 & 13 & 1,715 \\
\hline
\end{tabular}

a This table excludes one Latino patient who was mistakenly interviewed. 
Table 2

African Americans' First Interview Response and Nonresponse Rates by Patient Admitting Diagnosis by Interviewer-Patient Ethnic Match

\begin{tabular}{|c|c|c|c|c|c|c|c|c|c|}
\hline \multirow[b]{2}{*}{ Admitting diagnosis } & \multicolumn{2}{|c|}{ Completed } & \multicolumn{2}{|c|}{ Refused } & \multicolumn{2}{|c|}{ Discharged } & \multicolumn{2}{|c|}{ Other } & \multirow[b]{2}{*}{$N$} \\
\hline & $n$ & Row $\%$ & $n$ & Row $\%$ & $n$ & Row $\%$ & $n$ & Row \% & \\
\hline \multicolumn{10}{|c|}{ Interviewer of different ethnicity } \\
\hline Schizophrenia & 231 & 61 & 67 & 18 & 37 & 10 & 43 & 11 & 378 \\
\hline Mood disorder & 116 & 54 & 21 & 10 & 44 & 21 & 32 & 15 & 213 \\
\hline Total & 347 & 58 & 88 & 15 & 81 & 14 & 75 & 13 & 591 \\
\hline \multicolumn{10}{|c|}{ African American interviewer } \\
\hline Schizophrenia & 256 & 57 & 95 & 21 & 53 & 12 & 45 & 10 & 449 \\
\hline Mood disorder & 144 & 51 & 16 & 6 & 89 & 32 & 30 & 11 & 279 \\
\hline Total & 400 & 55 & 111 & 15 & 142 & 20 & 75 & 10 & 728 \\
\hline
\end{tabular}

Note. The overall $N$ does not sum to 1,333 because of missing data. 